\title{
Periosteal Layer of the Dura Mater
}

National Cancer Institute

\section{Source}

National Cancer Institute. Periosteal Layer of the Dura Mater. NCI Thesaurus. Code C33306.

The superficial layer of the cranial dura mater that lines the inner surface of the skull and functions as its periosteum. 